\title{
Associação entre a morfologia de trincas de pisos cerâmicos e seu comportamento mecânico em fratura
}

\section{(Association between crack morphology of ceramic floor tiles with their mechanical behavior in fracture)}

\author{
E. S. Fonsecal, F. G. Tavares ${ }^{1}$, T. N. R. Sousa ${ }^{1}$, W. A. Morais ${ }^{1,2}$ \\ ${ }^{1}$ UNISANTA, Rua Cesário Mota 08, Santos, SP 11045-040 \\ ${ }^{2}$ INSPEBRAS, Av. do Trabalhador 1446, Praia Grande, SP, Brasil 117250-000 \\ enirfonseca@gmail.com,gonzalezzft@gmail.com,thyrson@uol.com.br,willyank@unisanta.br
}

\begin{abstract}
Resumo
Este trabalho analisou os dados obtidos a partir de ensaios a flexão em um lote de 40 corpos de provas de placas cerâmicos, preparados da mesma forma de acordo com a norma brasileira de ensaios NBR 13818:1997. Os dados de resistência à flexão obtidos nos testes, na forma de curvas tensão versus flexão, foram avaliados estatisticamente. Análises estatísticas detalhadas mostraram dois subgrupos diferentes de comportamento mecânico apresentado pelos corpos de prova cerâmicos testados. A análise fractográfica de amostras representativas destes dois grupos indicaram diferenças morfológicas relevantes nas fraturas obtidas destas amostras durante o ensaio. Essas diferenças puderam ser associadas com o comportamento mecânico apresentados pelas amostras analisadas.
\end{abstract}

Palavras-chave: placa cerâmica, resistência mecânica, fractografia.

Abstract

This study analyzed data obtained from bending tests of a batch of 40 samples of ceramic tiles, identically prepared in according to the Brazilian test standard NBR 13818: 1997. The Flexural or bending strength data obtained from the tests, in the form of stress vs. deflection curve, were statistically evaluated. Detailed statistical analysis showed two different subgroups of mechanical behavior exhibited by the ceramic samples tested. The fractographic analysis of representative samples from these two groups showed significant morphological differences in the fracture of these samples obtained during test. These differences could be associated with the mechanical behavior exhibited by analyzed samples.

Keywords: ceramic plate, mechanical strength, fractography.

\section{INTRODUÇÃO}

Apesar da amplitude de tipos e possibilidades de uso, os materiais cerâmicos possuem uma limitada capacidade de deformação plástica na maioria das suas aplicações. Esta característica mecânica torna estes materiais particularmente sensíveis à presença de descontinuidades internas, cuja distribuição, tamanho e geometria desta forma acabam determinando a resistência final do material. Devido ao caráter probabilístico desta dependência, o físico sueco Wallodi Weibull desenvolveu um tratamento estatístico publicado em seu clássico artigo "Statistical Theory of The Strength of Materials" [1] como forma de predizer o comportamento à fratura de materiais que apresentem um comportamento puramente elástico. A distribuição de Weibull vem sendo empregada com sucesso na caracterização da resistência à fratura de materiais frágeis [2] assim como na descrição do comportamento em fadiga de materiais metálicos, sendo também empregada em análises de falhas [3].

Ensaios mecânicos para a caracterização de materiais estão associados, em maior ou menor grau, a imprecisões no que diz respeito à obtenção dos valores das propriedades mensuradas [4]. A sensibilidade dos materiais cerâmicos à presença de descontinuidades internas e a eventuais heterogeneidades na aplicação dos esforços mecânicos [2] torna relevante o uso de ferramentas estatísticas para a descrição dos resultados obtidos a partir de materiais cerâmicos testados mecanicamente. Tais ferramentas permitem identificar resultados consistentes ou indicar, com um determinado grau de precisão estatística, a existência de diferenças entre os materiais, fabricantes, lotes ou amostras a partir dos resultados obtidos. Entretanto esta avaliação não indica qual é a causa da diferença entre os resultados mensurados.

Por outro lado, a superfície gerada quando um material é fraturado possui características morfológicas específicas que permitem empregar a descrição topográfica desta fratura na compreensão de como esta foi formada [5]. Esta avaliação é feita através da fractografia, termo originado da união das palavras "fractus"(fratura) e "grapho" (tratamento descritivo, desenho) do latim. Através da fractografia é possível inferir, entre outras informações, por exemplo, o sistema de tensões 
que produziu a fratura, o local onde a fratura foi iniciada, a intensidade e a variação no carregamento mecânico aplicado [6]. Objetiva-se, com isso, associar o mecanismo de fratura presente com a história de utilização à qual o componente e/ ou estrutura foi submetido [5].

Neste trabalho foi utilizada a análise estatística de Weibull para avaliar a resistência mecânica de placas cerâmicas empregadas para revestimento. Um lote homogêneo de 40 amostras dessas placas foi testado através de ensaio de flexão a três pontos e os valores de resistência mecânica e as curvas tensão versus flexão obtidos foram avaliados. A distribuição de Weibull conseguiu descrever satisfatoriamente os valores da resistência mecânica final das placas cerâmicas testadas [6]. Porém a evolução do processo de fratura das placas testadas revelou a presença de dois subgrupos de comportamentos, cuja existência foi estatisticamente comprovada pela análise da evolução dos valores da carga suportada pelas placas em níveis pré-estabelecidos de deformação antes da ruptura. A análise fractográfica apontou características morfológicas específicas para cada um dos dois subgrupos identificados indicando um distinto comportamento nos ensaios, embora pertencessem ao mesmo conjunto e lote de amostras cerâmicas.

\section{MATERIAIS E MÉTODOS}

Foram preparados 40 corpos de provas oriundos de um mesmo lote e caixa de pisos cerâmicos de fabricação nacional com as seguintes características técnicas: piso semi-poroso esmaltado (tipo G), fabricado por prensagem com faixa de absorção de água de 6 a 10\% (BIIb). Os pisos apresentam alto nível de resistência química para álcalis em baixa concentração (LA) e pertencem à classe de resistência à abrasão superficial PEI (Porcelain Enamel Institute) 4, indicado para uso residencial interno e externo assim como a ambientes comerciais de tráfego médio. As placas são do padrão de superfície extra (A), no qual $95 \%$ das placas não apresentarem defeitos visíveis na distância de até $1 \mathrm{~m}$. Dimensões nominais das peças: $298 \mathrm{~mm}$ x $298 \mathrm{~mm}$ x 7,8 mm.

Os corpos de prova, ilustrados na Fig. 1a, foram cortados com uma serra diamantada com lubrificação a água no comprimento de $298 \mathrm{~mm}$; largura $148 \mathrm{~mm}$ e espessura 7,8 mm, conforme mostrado na Fig. 1b. Após a operação de corte, as amostras foram tratadas em uma estufa Inspebras ${ }^{\circledR}$ [7], ilustrada na Fig. 2a, conforme norma de ensaios NBR 13818:1997 [8]. Após concluído um ciclo de secagem a $110{ }^{\circ} \mathrm{C}$ durante $26 \mathrm{~h}$, as amostras foram resfriadas até a temperatura ambiente em um dessecador, para impedir o retorno da umidade. Os ensaios foram realizados dentro de um intervalo de uma hora após a retirada das amostras do dessecador para impedir a reabsorção de umidade.

Os corpos de prova preparados foram testados em flexão a três pontos em uma máquina universal de ensaios eletromecânica e microprocessada EMIC DL5000, Fig. 2b. Foi empregando o software Tesc 3.01 para o controle e obtenção dos dados oriundos dos ensaios.

As dimensões do dispositivo de dobramento, pontos de

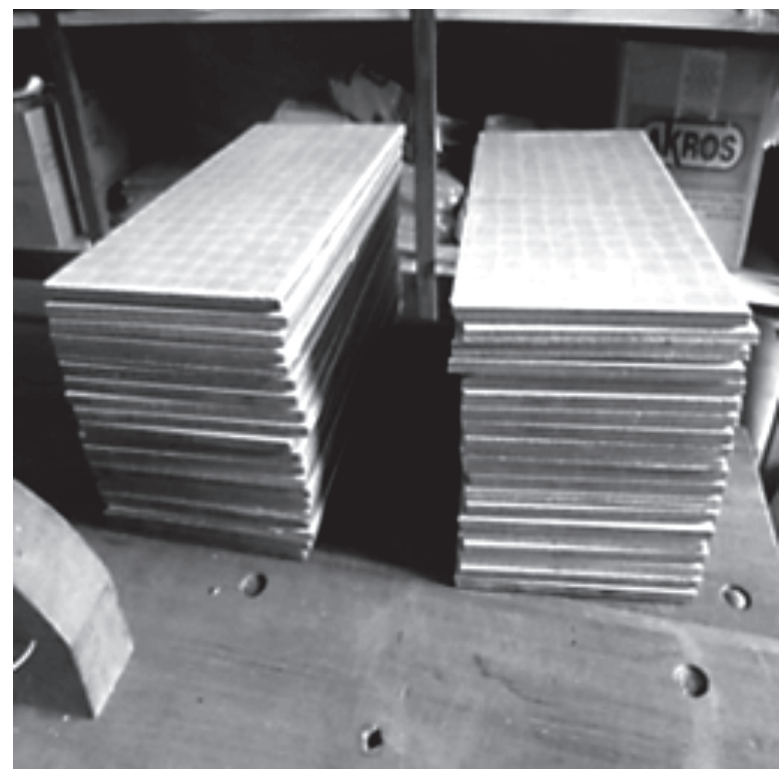

(a)

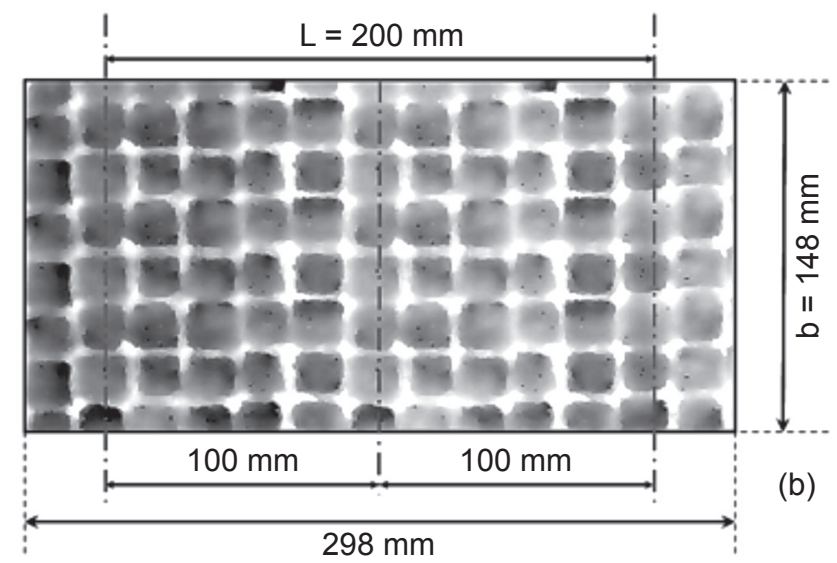

Figura 1: Corpos de prova empregados nos ensaios: a) aspecto visual das amostras; b) dimensões das amostras e posicionamento dos 3 pontos (linhas) de aplicação de carga.

[Figure 1: Samples employed on tests: a) visual aspect of the samples; b) sample dimensions and positioning of the three points (lines) of load application.]

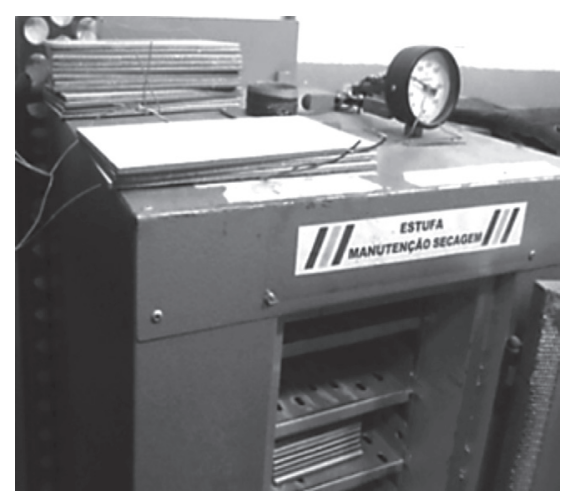

(a)

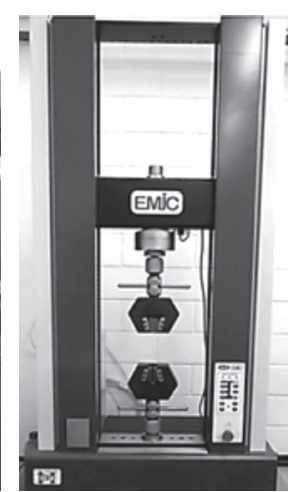

(b)
Figura 2: Equipamentos empregados: a) estufa de secagem; b) máquina de testes universal (tração e compressão).

[Figure 2: Employed equipments: a) drying oven; b) universal testing machine (tension and compression).] 
apoio e posicionamento na máquina universal de ensaios foram adotados em conformidade com a norma de ensaios NBR 13818:1997 [8] e estão ilustrados na Fig. 3a. A tensão de flexão aplicada durante o ensaio de flexão foi calculada conforme a equação $\mathrm{A}$, com base na força registrada pela célula de carga da máquina, mostrada na Fig. 3 b.

$$
\mathrm{MRF}=\frac{3 \cdot \mathrm{F} \cdot \mathrm{L}}{2 \cdot \mathrm{b} \cdot \mathrm{e}^{2}}
$$

na qual $M R F$ é o módulo de resistência à flexão, $F$ a força aplicada em Newton, $L$ a distância da barra de apoio em $\mathrm{mm}$, $b$ a largura do corpo de prova ao longo da ruptura em ms e $e$ mínima espessura do corpo de prova em $\mathrm{mm}$.

As 40 amostras de pisos cerâmicos foram numeradas de forma aleatória e posteriormente divididas em 3 grupos. O primeiro grupo (G1), composto por 3 corpos de prova, foi empregado para fazer ajustes no procedimento de realização dos ensaios e na velocidade da máquina de teste. O segundo grupo (G2), considerado o grupo principal, foi composto por 34 corpos de provas que foram empregados
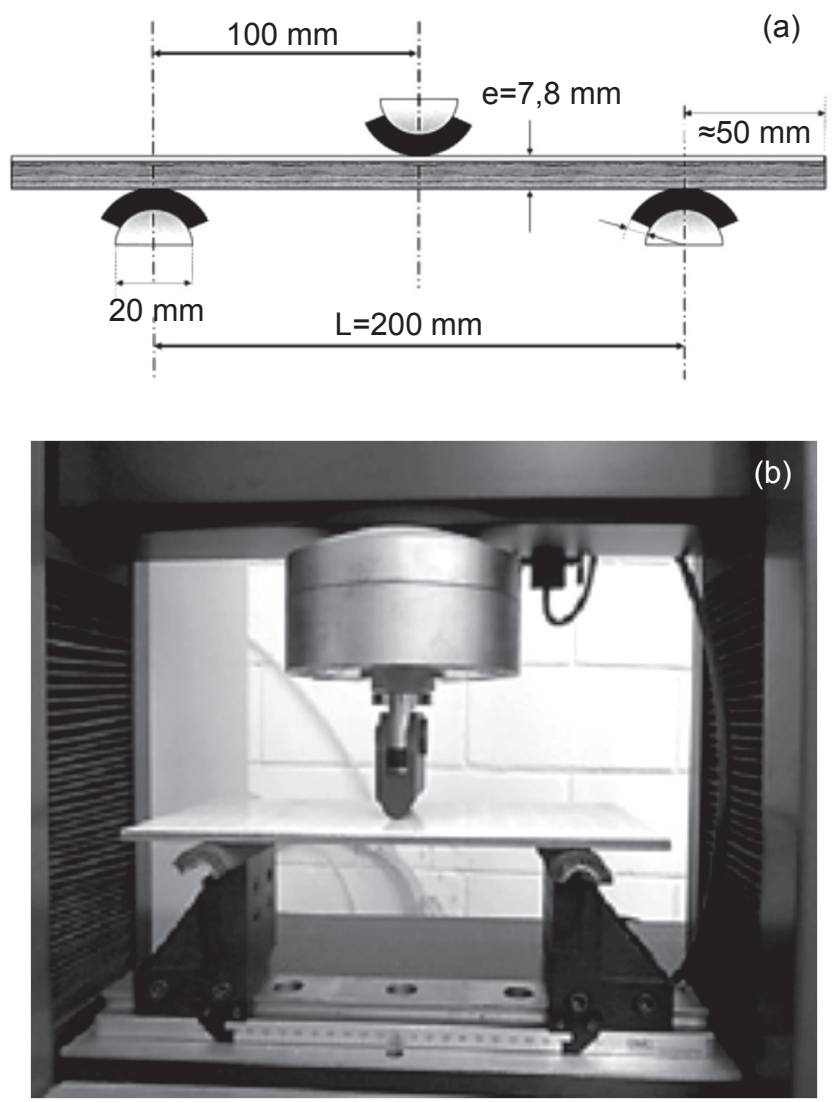

Figura 3: Montagem dos ensaios mecânicos: a) dimensões da amostra e dos pontos de apoio; b) posicionamento de uma amostra cerâmica no dispositivo de flexão a três pontos, neste caso o apoio superior foi trocado para facilitar a visão da amostra.

[Figure 3: Setup of the mechanical tests: a) dimensions of the samples and the support points; b) positioning a ceramic sample in the three-point device bending, in this case the upper support was changed to facilitate sample view.] para gerar os dados analisados neste trabalho. As últimas 3 amostras, que compuseram o terceiro e último grupo (G3), foram submetidas à imersão total em água por 30 min para verificar o grau de influência máxima da umidade nos resultados.

A norma NBR 13818:1997 [8] exige uma taxa de carregamento de $1 \pm 0,2 \mathrm{MPa} / \mathrm{s}$, mas o teste com as três amostras do primeiro grupo (G1) mostrou que a adoção desta taxa limitaria a quantidade de pontos a serem obtidos durante os testes. Além disso, os corpos de prova apresentaram dois regimes de dependência entre a tensão medida em função da flexão induzida pela máquina, o que exigiria a troca de velocidade do carregamento ao longo dos testes. Devido a estas questões, adotou-se uma velocidade fixa de deslocamento do travessão da máquina igual à $1 \mathrm{~mm} / \mathrm{min}$. Esta prática levou à obtenção de uma taxa de carregamento média de $0,231 \pm 0,01 \mathrm{MPa} / \mathrm{s}$.

Os ensaios com as 34 amostras do segundo grupo (G2) foram executados em uma única sequência ao longo de 2 $\mathrm{h}$ de trabalho com os mesmos dispositivos e parâmetros de ensaio para evitar que modificações nas condições do ensaio gerassem resultados variáveis. Além disso, antes do ensaio, cada amostra foi inspecionada visualmente para detectar possíveis avarias ou fraturas que inviabilizassem a representatividade dos resultados obtidos no seu teste.

Os resultados foram avaliados por meio de uma análise exploratória dos dados (AED) [9], especialmente focada na detecção de valores atípicos. Esses valores, conhecidos como outliers [10], quando ocorrem e possuem uma causa identificável, devem ser retirados do conjunto de dados, de forma a ser obtida uma análise estatística consistente com os dados restantes. Neste trabalho a identificação de outliers foi realizada pelo emprego de gráficos Box-Plot (caixa de bigodes) e pelo teste da razão de Dixon [11]. O Box-Plot é uma ferramenta gráfica que permite visualizar outliers, sua localização, dispersão e simetria independentemente da forma da distribuição do conjunto de dados [12]. Já o teste da razão de Dixon, que é um teste muito empregado para pequenas amostras, considera o grau de diferença entre o valor suspeito e o valor mais próximo. No teste de Dixon, outliers são identificados pela razão Q. Para um determinado nível de significância (p), comparam-se os valores críticos tabelados por Dixon com o valor de Q obtido: se o valor de $\mathrm{Q}$ for menor o valor não é outlier. Para amostras com mais de 14 membros, a razão de Dixon (Q) é calculada através da equação B:

$$
\mathrm{Q}=\mathrm{r}_{22}=\frac{\mathrm{x}_{3}-\mathrm{x}_{1}}{\mathrm{x}_{(\mathrm{n}-2)}-\mathrm{x}_{1}}
$$

na qual $x$ é o valor da variável mensurada, resistência mecânica (MPa) ou flexão máxima $(\mathrm{mm})$ da amostra cerâmica, ordenada do valor mais baixo $\left(x_{1}\right)$ até o valor mais elevado $\left(x_{n}\right)$ do conjunto de $n$ testes $(n>14)$.

Gráficos de probabilidade acumulada, que servem de base para verificar a distribuição normal dos dados foi outra 
forma de avaliação empregada neste trabalho para avaliar os dados obtidos. A normalidade dos dados também foi avaliada pelo parâmetro de Anderson-Darling (AD) e pelo valor-P de distribuição de probabilidade acumulada. Valores elevados de $\mathrm{AD}$ e baixos resultados do valor-P indicam uma distribuição não normal (gaussiana).

Particularmente, os resultados da resistência final das placas cerâmicas foram analisados pela distribuição de Weibull por meio do software Minitab ${ }^{\circledR}$ 17.1.0, sendo determinados os parâmetros da função de probabilidade de Weibull, representada pela equação $\mathrm{C}$, pelo método de Kaplan e Meier:

$$
\mathrm{P}(\mathrm{x})=1-\exp \left[-\left(\frac{\mathrm{x}-\mathrm{x}_{\mathrm{u}}}{\mathrm{x}_{0}}\right)^{\mathrm{m}}\right]
$$

na qual $x_{0}$ é o fator de escala, que é o valor característico da distribuição, que neste caso representa a resistência mecânica típica; $m$ é o parâmetro de forma da distribuição, mais conhecido como coeficiente de Weibull; $x_{u}$ é denominado parâmetro de localização, que é o menor valor característico da variável, no caso a resistência.

\section{RESULTADOS E DISCUSSÃO}

Os resultados dos valores de resistência mecânica à flexão, calculados pela equação $\mathrm{A}$, para as 34 amostras do grupo G2 estão sumarizados na Tabela I. Os valores empregados para o teste de Dixon estão mostrados na Tabela II. Nos dados obtidos, não foram detectados nenhum outlier, nem pelo uso dos Box Plot, nem através do teste de Dixon, dentro de um nível de significância de 5\%. Isso indica que

Tabela I - Resultados obtidos após ensaios com as amostras cerâmicas do grupo G2 (CP 4 ao 37).

[Table I - Results obtained after testing with the samples from group $\mathrm{G} 2$ (Sample 4 to 37).]

\begin{tabular}{ccccc}
\hline $\mathrm{CP}$ & $\mathrm{t}(\mathrm{s})$ & $\begin{array}{c}\boldsymbol{\delta}_{\text {máx }} \\
(\mathrm{mm})\end{array}$ & $\begin{array}{c}\mathrm{F}_{\text {máx }} \\
(\mathrm{N})\end{array}$ & $\begin{array}{c}\boldsymbol{\sigma}_{\text {max }} \\
(\mathrm{MPa})\end{array}$ \\
\hline 4 & 77,8 & 1,29641 & 498,16 & 16,60 \\
5 & 87,2 & 1,45337 & 612,12 & 20,39 \\
6 & 80,8 & 1,34720 & 592,58 & 19,74 \\
$\ldots$ & $\ldots$ & $\ldots$ & $\cdots$ & $\cdots$ \\
35 & 72,3 & 1,20453 & 533,97 & 17,79 \\
36 & 83 & 1,38278 & 556,77 & 18,55 \\
37 & 80,8 & 1,34722 & 524,21 & 17,47 \\
\hline
\end{tabular}

os cuidados tomados na preparação, acondicionamento e inspeção das amostras e durante a execução dos ensaios conseguiram evitar a dispersão de resultados obtidos até este nível de significância. Desta forma, os dados obtidos são suficientemente consistentes e susceptíveis para serem analisados com maior precisão pela distribuição de Weibull (equação C).

Utilizando os dados obtidos das 34 amostras testadas do Grupo G2, foram obtidos os seguintes valores para os três parâmetros de Weibull: módulo $\mathrm{m}=2,016$; o fator de escala $\mathrm{x}_{0}=2,981$ e valor de posição, $\mathrm{x}_{\mathrm{u}}=15,74$. O gráfico da Fig. 4 ilustra a distribuição de dados obtida e o gráfico da Fig. 5 ilustra as curvas tensão versus flexão para as 34 amostras analisadas pela distribuição de Weibull.

Apesar dos resultados obtidos da carga e flexão máximas no momento da fratura das amostras apresentarem um comportamento normal e ausência de outliers, as curvas tensão x flexão, obtidas ao longo dos testes, apresentam uma nítida tendência de agrupamento em torno de duas regiões, como mostrado na Fig. 5. Nestes grupos, denominados G2A e G2B, a diferença ocorre nos valores apresentados de resistência (tensão) apresentada pelas placas cerâmicas para um mesmo nível de flexão aplicado. Para analisar melhor esta característica, foram avaliados os valores de resistência mecânica apresentados pelas placas cerâmicas em níveis de flexão equidistantes e homogeneamente distribuídas ao longo dos ensaios.

A Fig. 6 apresenta as distribuições acumuladas de resistência em quatro níveis de flexão: 0,$60 ; 0,80 ; 1,00$ e $1,20 \mathrm{~mm}$. Os maiores valores de Anderson-Darling obtidos para os níveis de

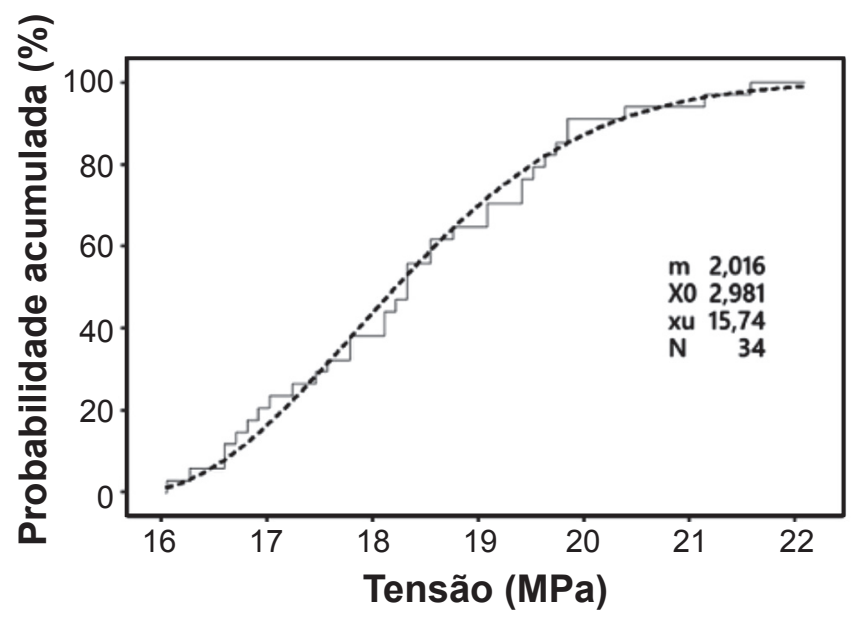

Figura 4: Gráfico da função de distribuição cumulativa (FDC) Weibull para os dados obtidos.

[Figure 4: Graph of the Weibull cumulative distribution function (CDF) for obtained data.]

Tabela II - Resultados obtidos após ensaios com as amostras cerâmicas do grupo de amostras G2 (CP 4 ao 37). [Table II - Results obtained after testing with the samples from sample group G2 (Sample 4 to 37).]

\begin{tabular}{ccccccccc}
\hline Variável & $\mathrm{N}$ & $\mathrm{x}_{1}$ & $\mathrm{x}_{2}$ & $\mathrm{x}_{3}$ & $\mathrm{x}_{(\mathrm{n}-2)}$ & $\mathrm{x}_{(\mathrm{n}-1)}$ & $\mathrm{x}_{\mathrm{n}}$ & $\mathrm{r}_{22}$ \\
\hline$\sigma(\mathrm{MPa})$ & 34 & 16,1 & 16,3 & 16,6 & 20,4 & 21,2 & 21,6 & 0,125 \\
$\delta(\mathrm{mm})$ & 34 & 1,197 & 1,198 & 1,199 & 1,454 & 1,498 & 1,513 & 0,007 \\
\hline
\end{tabular}




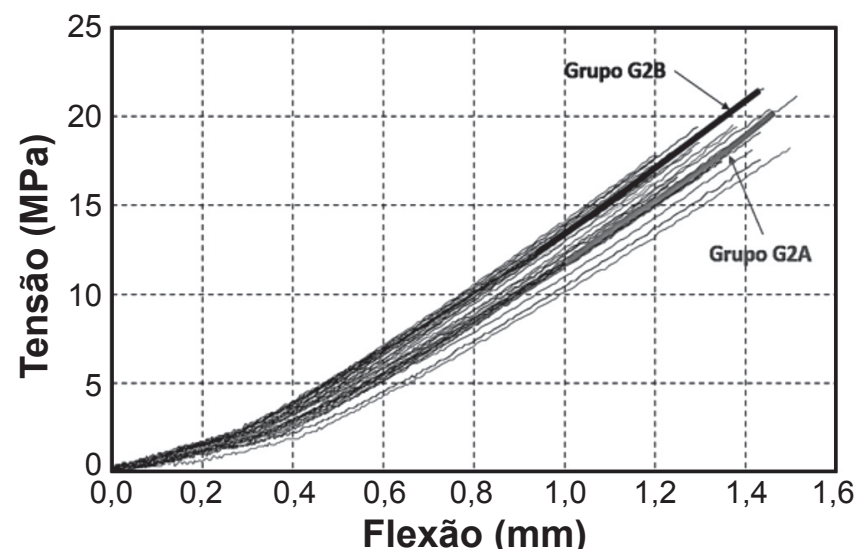

Figura 5: Curvas de tensão vs. flexão obtidas a partir das 34 amostras do grupo G2.

[Figure 5: Curves of tension vs. bending obtained from the 34 samples of $G 2$ group.]

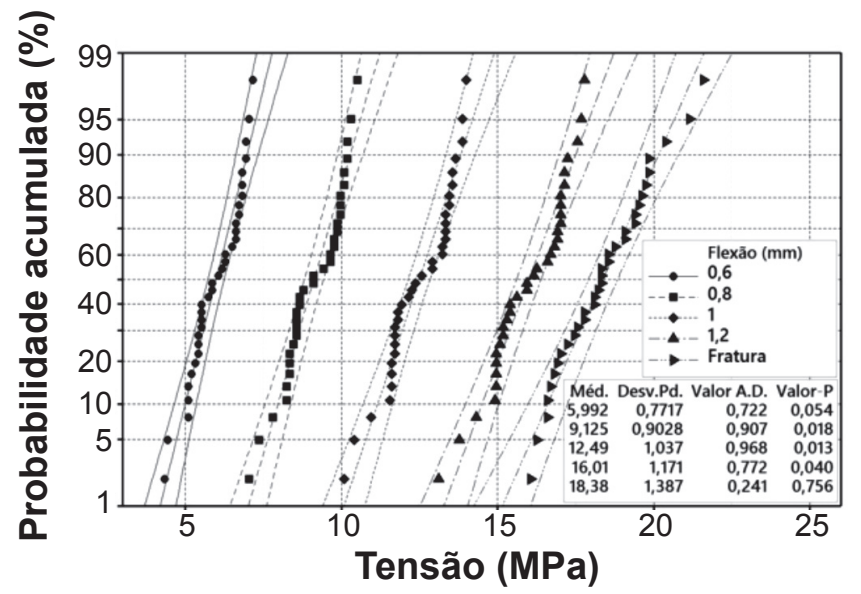

Figura 6: Distribuição de probabilidade do nível de resistência apresentada pelas 34 amostras do grupo G2 para 4 níveis de flexão e na fratura.

[Figure 6: Probability distribution of the strength displayed by 34 samples of group G2 for 4 levels of bending and on fracture.]

0,80 e $1,00 \mathrm{~mm}(0,907$ e 0,968 , respectivamente) indicam que a dispersão dos valores de resistência nestes níveis de flexão é maior do que a observada em fratura $(\mathrm{AD}=0,241)$.

Pelos dados obtidos, observou-se que as amostras 4, 27, 31 e 36 apresentaram valores de resistência pertencentes ao grupo G2A, de menor resistência, em pelo ao menos três níveis de deformação. Por outro lado, as amostras 6,25 e 34 foram as mais representativas do grupo G2B pela mesma razão. A Fig. 7 apresenta o aspecto visual das amostras 4 (G2A) e 34 (G2B), a linha de fratura foi ressaltada para demonstrar a diferença de aspecto. A Fig. 8 apresenta a comparação entre as geometrias das fraturas apresentadas pelas placas mencionadas anteriormente $(4,27,31$ e 36 G2A e 6, 25 e 34 - G2B).

Observando as representações das trincas das placas representativas do grupo G2A (menor resistência) e do grupo G2B (maior resistência), pode-se notar que as trincas das amostras do grupo G2A apresentam uma maior variação

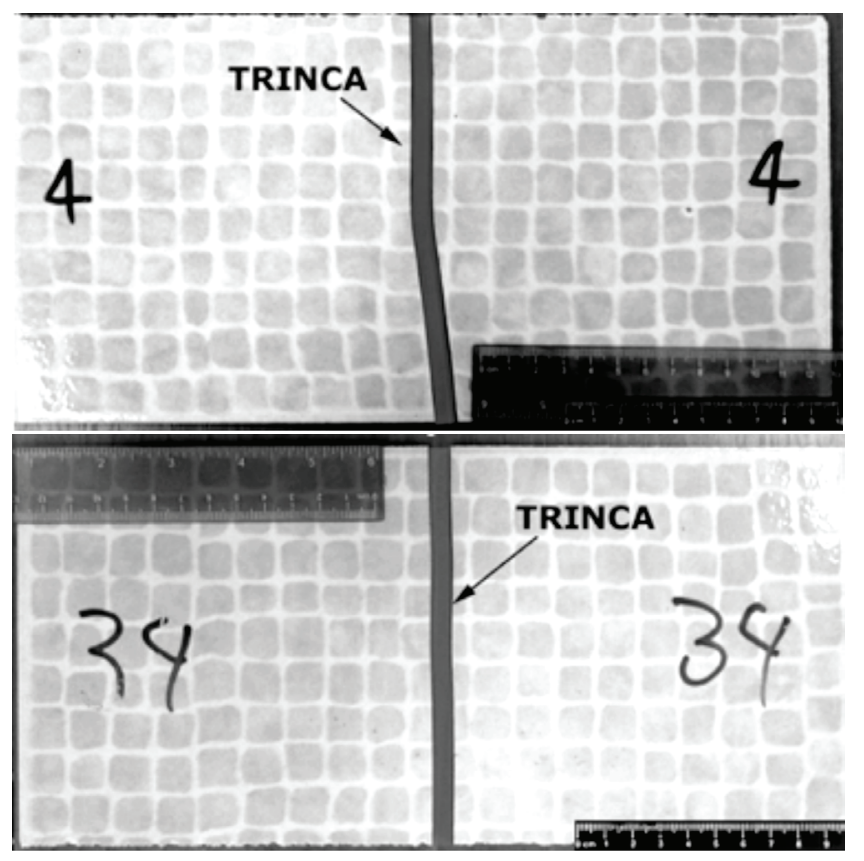

Figura 7: Aspecto da fratura final dos corpos de prova números 4 e 34, representativos dos grupos G2A e G2B, respectivamente.

[Figure 7: Final fracture aspect of the samples \#4 and \#34, representative of groups $G 2 A$ and $G 2 B$, respectively.]

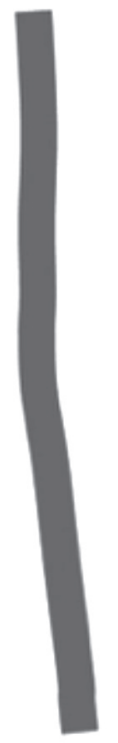

04

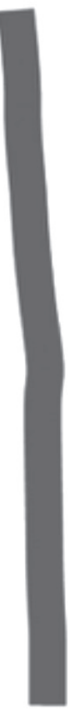

27

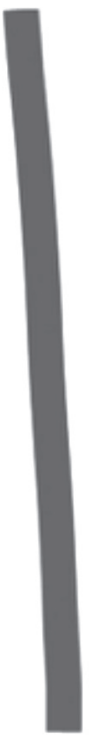

31

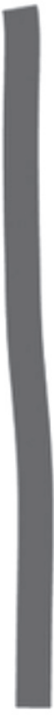

36

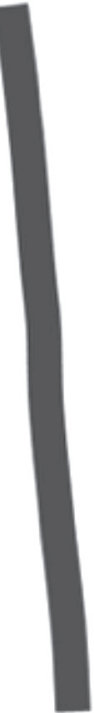

06

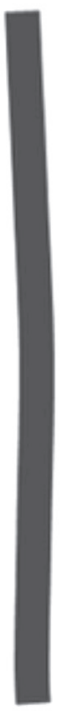

25

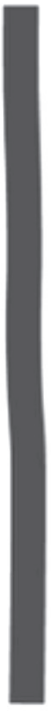

34
Figura 8: Geometria das trincas apresentadas pelos corpos de prova representativos dos grupos $\mathrm{G} 2 \mathrm{~A}(04,27,31$ e 36$)$ e $\mathrm{G} 2 \mathrm{~B}$ (06, 25 e 34).

[Figure 8: Crack geometries shown by representative samples of G2A group (04, 27, 31 and 36) and $G 2 B(06,25$ and 34).]

na sua linha de propagação. Isso indica que dentro da placa, uma maior e mais intensa fonte de nucleação de trincas estava presente, já liberando energia elástica ao longo do ensaio de dobramento. Esta fonte pode ser associada à incrustação de outros materiais, originados da matéria prima, ou pode ter sido resultado de uma má moagem, mistura ou queima das placas cerâmicas. Uma avaliação em 
lupa estereoscópica revelou que as trincas das amostras do grupo G2A apresentam-se mais recartilhadas do que as do grupo G2B. Uma caracterização detalhada provavelmente indicaria a diferença entre estas duas condições, porém esta caracterização está fora do escopo deste trabalho e deverá ser feita em um estudo posterior.

\section{CONCLUSÕES}

Ensaios de flexão a três pontos com 34 corpos de prova, em conformidade com a norma NBR 13818:1997, porém com uma taxa de carregamento de $0,231 \mathrm{MPa} / \mathrm{s}$, apresentaram um comportamento em fratura bastante similar. Análises estatísticas realizadas com os valores obtidos de tensão e flexão máximos obtidos em fratura indicam que estes valores apresentam um comportamento estatisticamente representativo, sem a ocorrência de outliers. Porém, observando-se mais criteriosamente os dados obtidos, representados pelas curvas tensão versus flexão, pode-se perceber que as mesmas amostras apresentam dois tipos de comportamento ao longo do ensaio, apresentando valores de resistência mecânica, para um mesmo nível intermediário de flexão, estatisticamente diferentes. Analisando as superfícies de fratura, pode-se perceber que as amostras pertencentes ao grupo de menor resistência apresentam formato mais irregular e recartilhado, indicando um processo de evolução de danos diferente das amostras do outro grupo. O tipo de comportamento observado aponta o cuidado que se deve ter com o teste de materiais cerâmicos, pois resultados mecanicamente similares podem estar associados a comportamentos diferentes, o que pode ser verificado por uma análise mais detalhada dos dados registrados ou das superfícies de fratura obtidos.

\section{AGRADECIMENTOS}

Os autores agradecem ao apoio dos técnicos da UNISANTA e da INSPEBRAS, que muito auxiliaram na preparação, execução e acompanhamento dos ensaios laboratoriais e de campo.

\section{REFERÊNCIAS}

[1] W. A. Weibull, "A Statistical Theory of The Strength of Materials", Ingenjörs vetenskaps akademiens Handlingar. 151 (1939) 5-45.

[2] Y. Wang, Y. C. Chan, Z. L. Gui, D. P. Webb, L. T. Li, "Application of Weibull distribution analysis to the dielectric failure of multilayer ceramic capacitors", Mater. Sci. Eng. B. 47 (1997) 197-203.

[3] F. J. Imasava, M. T. Gouvêa, C. Xavier, S. Rodrigues, Anais do $33^{\circ}$ Cong. Bras. Ceram., Serra Negra, SP (1989) 985-1000.

[4] F. G. Tavares, W. A. Morais, E. S. Fonseca, T. N. R. Souza, "Análise utilizando a distribuição de Weibull com dois e com três parâmetros para determinar a resistência mecânica a flexão de materiais cerâmicos", Unisanta Sci. Technol. 3, 2 (2014) 76-81.

[5] W. A. Morais, W. D. V. Lacerda, F. H. Vilela, E. S. Farias, "A Análise da falhas: Parte 2 - Morfologias básicas das superfícies de fratura", Siderurgia Brasil. 15, (2014), 20-22.

[6] G. M. Cândido, M. C. Rezende, M. V. Donadon, S. F. M. Almeida, "Fractografia de Compósito Estrutural Aeronáutico Submetido ao Ensaio de Tenacidade à Fratura Interlaminar em Modo II", Polímeros Ciência e Tecnologia. 24, 1 (2014) 65-71.

[7] INSPEBRAS, disponível em: http://www.inspebras. com.br/index.php, acesso em 17/05/2014.

[8] NBR 13818, "Placas cerâmicas para revestimento Especificação e métodos de ensaios", Associação Brasileira de Normas Técnicas (1997).

[9] NIST, "Statistics for Scientists \& Engineers: exploratory data analysis", disponível em http://www.itl.nist.gov., acesso em 20/08/2014.

[10] V. Barnett, T. Lewis, Outliers in statistical data, $3^{\text {th }}$ Ed., Wiley, Chichester, UK (1994) 584.

[11] W. J. Dixon, "Processing Data for Outliers", Biometrics 9, 1 (1953) 74-89.

[12] D. C. Hoaglin, F. Mosteller, J. W. Tukey, Análise exploratória de dados. Técnicas robustas: um guia, $1^{\mathrm{a}} \mathrm{Ed}$., Salamandra, Lisboa, Portugal (1992) 446.

(Rec. 16/03/2015, Ac. 08/05/2015) 